\section{Size constancy does not fail below half a degree}

As an observer's distance from an object varies, so does the visual angle subtended by the object at the observer's eye. One of the most reliable phenomena in psychology is that when distance information is available the perceived size of an object is nearly constant despite variations in visual angle, or retinal image size ${ }^{1}$. A corollary is that images of constant visual angle seem to vary in size with perceived distance (Emmert's law). This phenomenon ('size constancy') is only one part of the more general property of perception that one might call 'object constancy', embracing constancy of size, shape, depth, velocity, lightness, colour and so on. A recent report ${ }^{2}$ has claimed "that size constancy holds when image size is above half a degree, and breaks down when it is less". The claim, however, was based on demonstrations and anecdotal evidence, rather than experiment. We show experimentally that size constancy in the laboratory is equally good at target sizes above and below $\frac{10}{2}$.

The targets were single, black, vertical lines 1.2 arc min wide presented at three viewing distances $(114,228$ and $456 \mathrm{~cm})$ with four lengths $\left(\frac{1}{8}, \frac{1}{4}, \frac{1}{2}, 1 \mathrm{deg}\right)$. Each line was fixed in the centre of a white card subtending $5.25^{\circ}$ vertically by $3.75^{\circ}$ horizontally independent of viewing distance. Each card was placed at the appropriate position on a long laboratory bench on which papers and small objects were scattered. Viewing was binocular with full room lighting. Thus, distance information was abundant, but for a given target size (angle), the length and width of the line and of the frame in which it was set were of constant visual angle, independent of distance. The subject looked back and forth between the target and an oscilloscope screen placed at $228 \mathrm{~cm}$ distance, about $6^{\circ}$ to the right of the target. His task was to adjust the vertical separation between two small green dots on the screen until the separation 'appeared to match the size of the target'. Trials were presented in random order and the 12 conditions were repeated 3 times for each of the 10 subjects $(5$ male, 5 female).

The results (Fig. 1) show clearly that perceived length of a line of constant visual angle varies with distance in almost exactly the same way for all target sizes in the range $\frac{10}{8}-1^{\circ}$. Analysis of variance not surprisingly showed highly significant effects of both visual angle and viewing distance $(P \ll 0.001)$, but most importantly the interaction between distance and visual angle was not significant $\left(F_{6.29}=1.47, P>0.10\right)$. If size constancy were weaker or absent below half a degree the slope of the curves in Fig. 1 would be shallower or even flat for small target lengths. Our data strongly contradict any such effect. Constancy was not complete (this would be given by a slope of 1) but it was equally good for all target sizes.

In a second part of the experiment, an electronic flashgun was used to create the afterimage of a line $\frac{10}{4}$ long. The observer viewed the afterimage on a plain white card of constant visual angle at each of the three distances, and made length matches as in the first part of the experiment. A second or third exposure of the line was given if necessary to complete a total of nine trials. One subject reported no afterimage, but the data from the remaining nine (crosses in Fig. 1) were not significantly different from those obtained with a real line of $\frac{10}{4}\left(F_{1,8}=0.84\right.$, not significant).

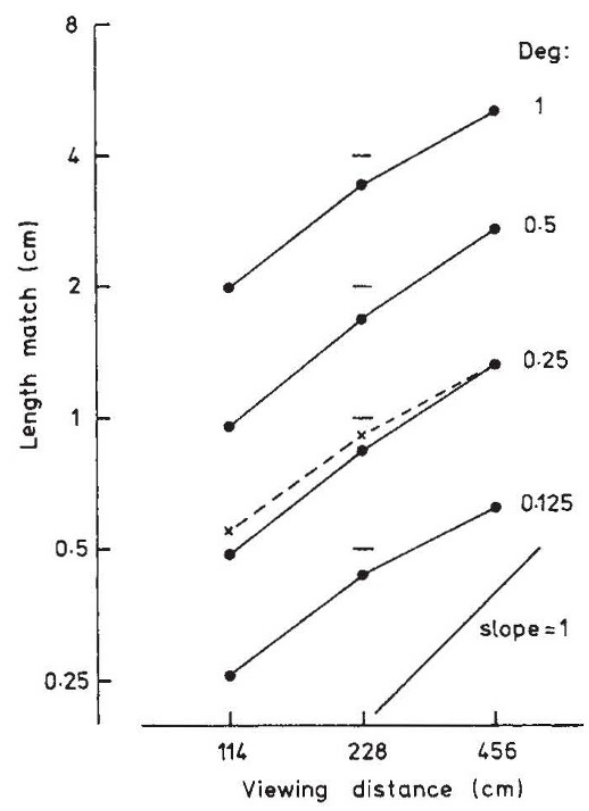

Fig. 1 Solid symbols show results averaged over 10 subjects who adjusted a pair of dots to match the lengths of lines subtending four different visual angles at three viewing distances. Crosses represent similar judgements (nine subjects) made with an afterimage $\frac{1}{4}$ long. Horizontal bars represent the ideal match when target and dots were at the same distance. A slope of 1 would represent perfect constancy, and zero slope would indicate judgements based purely on visual angle (no constancy). The average slope of regression lines fitted to the solid points was 0.70 , with no significant interaction between distance and visual angle.

Ross et al. ${ }^{2}$ reported that afterimages subtending less than $\frac{10}{2}$ did not seem to change size with 'projected' distance, and that the finding was robust in classroom demonstrations. We flashed a $2-\mathrm{cm}$ line to a group of 27 students, all of whom were more than $228 \mathrm{~cm}$ distant. The afterimage would thus be less than $\frac{1}{2}^{\circ}$ for all observers. They observed the image on their hands and then on a far wall. None reported no change of size, 19 said the image got bigger when further away, 2 said it got smaller and 6 reported no image. Ross $e t$ $a .^{2}$ also appeal to the reader's direct experience of the breakdown of constancy. Our own experience confirms our classroom demonstration, that small afterimages obey Emmert's law in the usual way. When we acted as subjects in the experiment, our data were virtually identical to those of Fig. 1.

Ross et al. ${ }^{2}$ remark that objects seen in the distance or from the top of a tall building seem 'small'. It may be that in these cases, and in their Fig. 1, information about the true distance is inadequate, or impoverished by poor resolution of detail. As a result, both distance and size would be underestimated ${ }^{1}$. The fact that many distant objects subtend less than $\frac{1}{2}^{\circ}$ may be quite incidental.

We conclude from our experiments and demonstrations that size constancy for both real targets and afterimages is just as good for small images as for large ones. Indeed, we should suffer very curious distortions in the perceived relative size of objects if this were not so. There seems to be no substance to the claim that size constancy fails below half a degree.

We thank the SRC for financial support.

\section{A. GEORGESON M. G. HARRIS}

University of Bristol, Department of Psychology, 8-10 Berkeley Square, Bristol BS8 1HH, UK

1. Rock, I. An Introduction to Perception, Ch. 2 (Macmillan, New York, 1975).

2. Ross, J., Jenkins, B. \& Johnstone, J. R. Nature 283, 473 (1980).

\section{Matters Arising}

Matters Arising is meant as a vehicle for comment and discussion about papers that appear in Nature. The originator of a Matters Arising contribution should initially send his manuscript to the author of the original paper and both parties should, wherever possible, agree on what is to be submitted. Neither contribution nor reply (if one is necessary) should be longer than 300 words and the briefest of replies, to the effect that a point is taken, should be considered. 